\title{
Lorenzen, Stefanie (2020). Entscheidung als Zielhorizont des Religionsunterrichts? Religiöse Positionierungsprozesse aus der Perspektive junger Erwachsener (Praktische Theologie heute I 74). Stuttgart: Kohlhammer. ISBN 978-3-I 7-038 I 54-4. 362 Seiten.
}

\author{
Rudolf Englert \\ Universität Duisburg-Essen (englertrudolf@gmail.com)
}

Die Entscheidungs- bzw. Positionierungsfähigkeit der Schüler*innen spielt eine wichtige Rolle für die Beschreibung des religionsunterrichtlichen Zielhorizonts. Das Bemühen um eine solche Fähigkeit ist sogar zu einem Identitätsmarker des konfessionellen Ansatzes geworden. Doch was wird unter einer Entscheidung bzw. unter einer Position im Feld des Religiösen eigentlich verstanden? Hierüber besteht weitaus weniger Klarheit, als man erwarten sollte. Diese offene Stelle ist der Ausgangspunkt der Untersuchung von Stefanie Lorenzen, einer in Saarbrücken vorgelegten Habilitationsarbeit.

Wie stellt sich eine „Entscheidung“ im Umgang mit Religion aus empirischer Sicht heute dar - also im Kontext eines Aufwachsens unter den Bedingungen von Säkularität und religiöser Pluralität? Aus der Befragung einschlägiger religionssoziologischer und psychologischer Befunde geht hervor: Wo sich eine „Eindeutigkeit der Selbstzuordnung zu ,typischen' Bestandteilen traditioneller Religiosität erkennen lässt" (29), ist dies häufig gerade keine Entschiedenheit aus persönlicher Überzeugung, sondern Konsequenz einer im Grunde kontingenten sozialisatorischen Einbindung. Wo dagegen eine persönliche Auseinandersetzung stattfindet, fällt oft gerade keine eindeutige Entscheidung, sondern wird „stattdessen der ,unentschiedene‘ Zwischenbereich gewählt“ (29). Es kommt „Zu situativ offenen, fluiden Formationen im Zwischenraum von Religiosität und Nicht-Religiosität“ (43). Diese seien, so Lorenzen, meist nicht das Ergebnis gedanklich erarbeiteter Zugehörigkeitsentscheidungen, sondern situativer Passungsreaktionen im Anschluss an die Frage: Was von all dem Religiösen passt zu mir? Solche Befunde ließen es geraten erscheinen, vom Begriff der Entscheidung als Zielformulierung religionspädagogischen Handelns Abstand zu nehmen.

Vor diesem Hintergrund wird eine Reihe religionspädagogischer Entwürfe (Schweitzer, Ziebertz, Dressler und Englert) daraufhin befragt, welche Rolle „Entscheidung“ als Zielperspektive darin jeweils spielt. Obwohl diese Entwürfe je etwas anders akzentuiert seien, verbinde sich mit ihnen, so Lorenzen, doch durchweg ein Verständnis religiöser Positionierung, „,das im Ergebnis nur auf ein ,Ja' oder ,Nein’ hinauslaufe“ (93). Damit werde der Breite möglicher religiöser Verortungen nicht angemessen Rechnung getragen. Dies gelte erst recht für ältere religionsdidaktische Ansätze wie die Evangelische Unterweisung, den hermeneutischen Religionsunterricht oder Nipkows Elementarisierungskonzept (302-324).

Der Verdacht, dass die Rede von „Entscheidung“ den bei Jugendlichen heute anzutreffenden Formen religiöser Positionierung nicht mehr angemessen sei, ist der Auslöser für die im Zentrum von Lorenzens Arbeit stehende empirische Studie. Deren Fragestellung lautet: „Wie lassen sich empirisch zu beobachtende religiöse Positionierungsprozesse theoretisch (angemessen) erfassen“ (124)? Klärung soll eine an der Grounded Theory orientierte qualitative Studie schaffen, in der 14 Interviews mit jungen Erwachsenen im Alter zwischen 18 und 26 Jahren ausgewertet werden.

Die zentralen theoretischen Kategorien, die Lorenzen aus der Auswertung dieser Interviews gewinnt, sind „aufgeladenes Wirkzentrum“ und „Verdichtung“. Damit spricht die Verfasserin die subjektive Erfahrung („Verdichtung“) einer Halt gebenden Größe („,aufgeladenes Wirkzentrum“) an. Es liegt nahe, dass auch Religion als ein aufgeladenes Wirkzentrum fungieren kann; wobei Lorenzen gerade herausfinden möchte, in welch verschiedenen Formen der Verdichtung Religion vom Einzelnen biografisch erfahren wird. Aus einer Vielzahl von Unterscheidungen entwickelt sie vier markante Muster, die sie an Fallbeispielen illustriert: 1. Die intim-okkasionelle Verdichtung, die in Richtung einer Stoßgebets-Religiosität geht; 2. Die intim-prinzipielle Verdichtung, die auf einem relativ sta- 
bilen Habitus individueller religiöser Orientierungssuche beruht (möglicherweise „ein Phänomen der Bildungselite“, 214); 3. Die sozial-extensive Verdichtung, die hauptsächlich in Gestalt von Partizipationserfahrungen gegeben ist (z. B. im Rahmen kirchlicher Verbandsarbeit); und schließlich 4. die sozial-intensive Verdichtung, die mit der Erfahrung religiöser bzw. weltanschaulicher Gleichgestimmtheit zusammenhängt.

Wie Religion in der Wahrnehmung Einzelner ,konstruiert' wird und in welcher Weise man sich dieser Religion gegenüber positioniert, hängt nach Lorenzen entscheidend davon ab, welche Verdichtungserfahrungen im Hintergrund stehen und ob diese Erfahrungen, was bei allen vier Mustern der Fall sein kann, das Subjekt gegenüber Religion eher öffnen oder verschließen. Religionspädagogisch komme es von daher darauf an, zunächst „möglichst umfassende Erfahrungsmöglichkeiten im Rahmen unterschiedlicher Ausprägungen von Religion“ (257) zu eröffnen und dann diese Erfahrungen daraufhin zu befragen, inwieweit Religion hier für den Einzelnen als „Halt im Leben“ erkennbar werde. Eine solche Wahrnehmung von Religion als persönlicher Ressource sei deutlich wichtiger als ihre Einschätzung als kognitiv überzeugendes Orientierungssystem (258f.).

Am Ende sind es vor allem drei Kriterien, an denen die Autorin ihre Empfehlungen zur Unterstützung religiöser Positionierungsprozesse im Religionsunterricht orientiert: 1. Einer Phase der Positionierung muss eine Phase religiöser Verdichtung bzw. religiöser Erfahrung vorausgehen; 2. Die zum Zweck religiöser Verdichtungen präsentierten „religiösen Gegenstände“ dürfen nicht nur aus einem einzigen religiösen Identifikationsraum stammen; 3 . Den Schüler*innen muss unter dem Kriterium „Was davon passt zu mir?“ ein breites Spektrum individueller Passungsreaktionen ermöglicht werden. Im Schnittpunkt dieser religionspädagogischen Anforderungen scheint das vor allem in England verbreitete Konzept "A Gift to the Child“ zu stehen. Hier sieht Lorenzen sich einen dritten Weg auftun „Zwischen den Optionen von ,konfessioneller' versus kulturwissenschaftlich-religionskundlicher Ausrichtung“ (345), einen Weg, der die Schüler*innen nicht mit Entscheidungszwängen überfordert.

Über die Studie von Stefanie Lorenzen lässt sich viel Gutes sagen: was die Relevanz des Themas angeht, die Qualität der theoretischen Auseinandersetzung mit einer Vielzahl von Konzepten aus diversen Wissenschaften, die wechselseitige Durchdringung theoretischer Perspektiven und empirischer Befunde oder das hoch entwickelte Differenzierungsvermögen der Autorin. Vor allem hat die Autorin ihre zentrale Intention: die „Weiterentwicklung des religionspädagogischen Positionierungsdiskurses“ (269), im theoretisch-hinführenden und im empirischen Teil der Arbeit auf einem beeindruckenden Niveau eingelöst. Zweifelhaft allerdings erscheint mir, was sie im abschließenden religionspädagogisch-praktischen Teil als „dritten Weg“ empfiehlt und in dem Konzept "A Gift to the Child“ exemplarisch realisiert sieht. Diese „Lösung“ der Positionierungsfrage legt sich von ihren eigenen Überlegungen und Befunden keineswegs so stark nahe, wie es die Autorin im Finale der Arbeit erscheinen lässt. Dies hat vor allem damit zu tun, dass der Gegenstand der empirischen Untersuchung: biografische Identifikationsprozesse im Leben junger Erwachsener, und der Gegenstand der religionsunterrichtlichen Empfehlungen: Positionierungen von Schüler*innen im Kontext des Religionsunterrichts, zwei sehr verschiedene Dinge sind. Positionierungsprozesse im Religionsunterricht zielen in der Regel ja nicht gleich auf die umfassende Frage nach der eigenen religiösen Identität, sondern meist viel spezifischer auf die Frage nach der eigenen Einschätzung bestimmter im Unterricht präsentierter Sichtweisen. Lorenzen spricht diese Differenz zwischen biografischem und schulischem Lernen zwar verschiedentlich an, trägt ihr bei ihren Überlegungen, wie religionsunterrichtliche Positionierungsprozesse am besten unterstützt werden können, aber nicht ausreichend Rechnung. Dies führt dazu, dass in den praktischen Empfehlungen Faktoren wie die kognitive Aktivierung der Schüler*innen, die Befragung religiöser Geltungsansprüche oder der Eigenanspruch religiöser Konzepte kaum eine Rolle spielen. 